Check for updates

Cite this: J. Mater. Chem. A, 2020, 8 , 2769

Received 6th August 2019

Accepted 22nd November 2019

DOI: $10.1039 / c 9 t a 08607 b$

rsc.li/materials-a

\title{
Engineering the conductive carbon/PEO interface to stabilize solid polymer electrolytes for all-solid- state high voltage $\mathrm{LiCoO}_{2}$ batteries $\uparrow$
}

\author{
Jianneng Liang, ${ }^{a}$ Yipeng Sun, ${ }^{a}$ Yang Zhao, ${ }^{a}$ Qian Sun, ${ }^{a}$ Jing Luo, ${ }^{a}$ Feipeng Zhao, ${ }^{a}$ \\ Xiaoting Lin, ${ }^{a}$ Xia Li, ${ }^{a}$ Ruying Li, ${ }^{a}$ Li Zhang, $^{b}$ Shigang Lu, ${ }^{b}$ Huan Huang ${ }^{\mathrm{c}}$ \\ and Xueliang Sun $(\mathbb{D}$ *a
}

\begin{abstract}
All-solid-state lithium batteries (ASSLBs) are promising energy storage devices for application in electric transportation and large-scale energy storage systems. Polyethylene oxide (PEO)-based solid polymer electrolytes (SPEs) are attractive solid-state electrolytes for ASSLBs due to their high ionic conductivity, light weight, and low cost. However, the low electrochemical oxidation potential window of PEO seriously restricts its implementation with high voltage cathodes for high-energy-density ASSLBs. Effective interfacial engineering between high voltage cathodes and SPEs can be a solution. Most of the reported conventional cathode protection approaches have been focused on building coating layers on active material particles, which, however, can be insufficient because the conductive carbon is able to accelerate the decomposition of SPEs. In this work, atomic layer deposition (ALD) coating on the electrode instead of active material particles realizes a unique method to protect the cathode/SPE interface. As a successful example, a thin ALD-derived lithium tantalate coating on the high-voltage $\mathrm{LiCOO}_{2}$ electrode demonstrated good compatibility with PEO-based SPEs, significantly enhancing the cycling performance of the ASSLBs. The inner mechanism is attributed to the fact that the protection of the conductive carbon/SPE interface helps reduce the electrochemical oxidation of PEO-based SPEs. This work shall give new insights for the interfacial engineering of high voltage cathodes and solid polymer electrolytes.
\end{abstract}

\section{Introduction}

All solid-state lithium batteries (ASSLBs) are promising candidates for application in electric vehicles (EVs) and other largescale energy storage systems due to their high energy density and enhanced safety. Feasible solid-state electrolytes (SSEs) are a key component to realize ASSLBs. Among all the solid-state lithium ion conductors, SSE systems including solid polymer electrolytes (SPEs), oxide-based SSEs, sulfide-based SSEs, and hybrid electrolytes received the most research interest. ${ }^{1-4}$ To realize the practical applications of ASSLBs, the following challenges of SSEs must be addressed: (i) high interfacial resistance, (ii) low ionic conductivity, (iii) narrow electrochemical window, and (iv) instability with electrodes. ${ }^{2,5,6}$

SPEs based on polyethylene oxide (PEO) and lithium salt complexes are promising candidates for ASSLBs, owing to their

${ }^{a}$ Department of Mechanical and Materials Engineering, University of Western Ontario, Canada.E-mail: xsun9@uwo.ca

${ }^{b}$ China Automotive Battery Research Institute Co., Ltd., China

${ }^{c}$ Glabat Solid-State Battery Inc., Canada

$\dagger$ Electronic supplementary information (ESI) available. See DOI: $10.1039 / \mathrm{c} 9 \mathrm{ta} 08607 \mathrm{~b}$ high ionic conductivity at elevated temperature and low interfacial resistance toward electrodes. More importantly, PEO is commercially available, low cost, light weight, and environmentally friendly. ${ }^{6}$ However, (1) the low ionic conductivity at room temperature,$^{6-8}(2)$ the susceptibility to lithium dendrites ${ }^{9}$ and (3) the instability at high voltage ${ }^{10}$ of PEO-based SPEs are the main issues that hinder their wide applications in ASSLBs.

Tremendous research efforts have been dedicated to address challenges (1) and (2) in recent years. For example, Cui's group enhanced the RT ionic conductivity of PEO-based SPEs from $10^{-7} \mathrm{~S} \mathrm{~cm}^{-1}$ to $4.4 \times 10^{-5} \mathrm{~S} \mathrm{~cm}^{-1}$ by in situ formation of nanosized $\mathrm{SiO}_{2}$ particle fillers in the PEO matrix. ${ }^{8} \mathrm{Hu}$ 's group created a 3-dimensional garnet-type SSE nanofiber network for PEO to form a hybrid electrolyte that has an ionic conductivity of $2.5 \times 10^{-4} \mathrm{~S} \mathrm{~cm}^{-1}$ at RT. ${ }^{11}$ Plenty of studies applying inorganic SSE fillers in the PEO matrix to enhance the RT ionic conductivity of PEO-based SPEs have also been reported. ${ }^{12,13} \mathrm{On}$ the other hand, many studies were reported to address the lithium dendrite formation problem in ASSLBs with PEO-based SPEs. ${ }^{11-14}$ For example, Zhao et al. prepared a LLZTO-PEO hybrid electrolyte for ASSLBs and they proposed that anions were tethered in the hybrid electrolyte by the polymer matrix and ceramic fillers, which results in a uniform distribution of 
space charges and lithium ions, thus, resulting in dendrite-free lithium deposition. ${ }^{\mathbf{1 4}}$

However, the issue of the instability of PEO-based SPEs at high voltage (challenge (3)) received little research attention. The instability of PEO at high voltage makes it challenging to couple with high voltage cathodes such as $\mathrm{LiCoO}_{2}$. Even though PEO-based SPEs show excellent stability with $\mathrm{LiFePO}_{4}$ cathode with charge voltage below $4 \mathrm{~V}\left(v s . \mathrm{Li} / \mathrm{Li}^{+}\right),{ }^{15}$ the limited theoretical energy density of $\mathrm{LiFePO}_{4}$ restrains its further application in future electric transportation. Alternatively, layer structured oxide cathodes such as $\mathrm{LiCoO}_{2}$ have a high specific capacity and high charge/discharge voltage, which makes them promising candidates for high-energy-density ASSLBs. Since the practical capacity of $\mathrm{LiCoO}_{2}$ is positively related to the charge cut-off voltage, a high charge cut-off voltage is required for highenergy-density applications. ${ }^{16}$ However, charge cut-off voltage over 4.5 V remains a challenge for PEO-based SPEs due to the decomposition of PEO-based SPEs at high voltage. ${ }^{17-19}$

One solution to enable PEO-based SPE coupling with high voltage $\mathrm{LiCoO}_{2}$ is interfacial engineering of SPEs and the $\mathrm{LiCoO}_{2}$ active material interface with coating layers. Coatings on $\mathrm{LiCoO}_{2}$ particles with $\mathrm{Al}_{2} \mathrm{O}_{3},{ }^{19} \mathrm{Li}_{3} \mathrm{PO}_{4},{ }^{18}$ poly(ethylcyanoacrylate) (PECA), ${ }^{20}$ and $\mathrm{Li}_{1.4} \mathrm{Al}_{0.4} \mathrm{Ti}_{1.6}\left(\mathrm{PO}_{4}\right)_{3}$ (LATP) ${ }^{21}$ have been reported to improve solid-state batteries performances. However, most of these previous studies only studied the coating effect on the active material/SPE interface while the conductive carbon/SPE interface is ignored. Unfortunately, conductive carbon has been proved to be able to accelerate the decomposition of SSEs including PEO-based SPEs, oxide-based SSEs and sulfide-based SSEs. ${ }^{10,22,23}$ Thus, the detrimental effect of conductive carbon on the PEO-based SPE cannot be neglected in high voltage ASSLBs. Therefore, systematic studies on the effects of coatings on active material particles versus on the whole electrodes (i.e. covering both active material and conductive carbon) are important to disclose the interfacial engineering mechanism and can provide us with helpful insights for future high-energy-density ASSLBs designs.
Herein, atomic layer deposition (ALD), as an emerging technique, which is capable of depositing uniform and conformal thin films with precise thickness control by selflimited chemical reactions, is chosen for realizing active material particle coating and whole electrode coating because the ALD process can be carried out at a low temperature compared to other chemical/physical deposition techniques such as chemical vapor deposition (CVD), physical vapor deposition (PVD), sol-gel methods, etc. Thus, ALD can be nondestructive to electrode components (cathode particles and conductive carbon). In this study, ALD derived lithium tantalate protective coatings are demonstrated to stabilize the interface between PEO-based SPEs and $\mathrm{LiCoO}_{2}$ electrodes at high voltage (4.5 V vs. $\mathrm{Li} / \mathrm{Li}^{+}$). Effects of coatings on the $\mathrm{LiCoO}_{2}$ particles, the whole electrode, and conductive carbon particles are compared. The results show that the coating on $\mathrm{LiCoO}_{2}$ particles cannot improve the performance of ASSLBs, while the coating on electrode sheets (coating on both $\mathrm{LiCoO}_{2}$ particles and carbon particles) shows significant enhancement in cycling performance, the same as the coating on conductive carbon particles. The working mechanism of the ALD coating is illustrated in Fig. 1. These results highlight that a suitable coating approach is critical in preventing the electrochemical oxidation of PEObased SPEs at high voltage for the stabilization of the high voltage performance of ASSLBs.

\section{Experimental section}

\section{Preparation of PEO-based SPEs}

PEO (M.W. 1000 000), $\mathrm{LiClO}_{4}$ (purity, 99.9\%) and garnet-type SSEs $\left(\mathrm{Li}_{6.4} \mathrm{La}_{3} \mathrm{Zr}_{1.4} \mathrm{Ta}_{0.6} \mathrm{O}_{12}\right.$, LLZTO) were dried in a vacuum oven before using. $0.24 \mathrm{~g}$ of LLZTO was added into $50 \mathrm{~mL}$ of acetonitrile (AN) and mixed by ultrasonication for $5 \mathrm{~h}$. Then, $1.2 \mathrm{~g}$ of PEO and $0.19 \mathrm{~g}$ of $\mathrm{LiClO}_{4}$ were added into the mixture and stirred overnight at room temperature. This homogeneous mixture was cast onto a Teflon substrate and dried at room temperature overnight to slowly evaporate the AN, followed by (a) Bare cathode/SPE interface

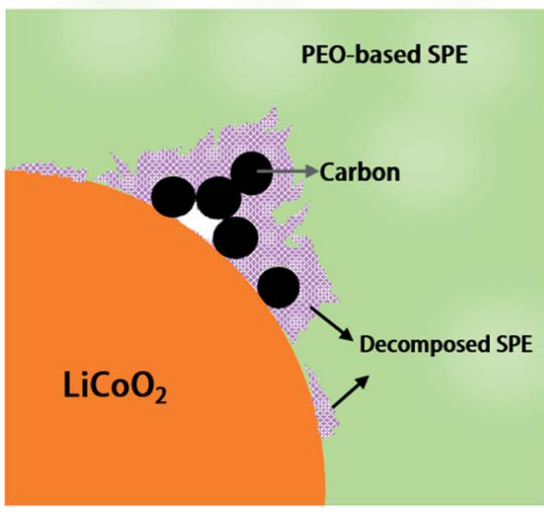

(b) Particles coating cathode/SPE interface

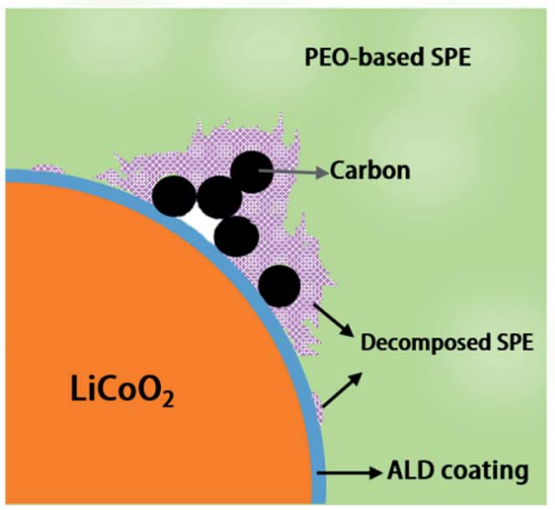

(c) Electrode coating cathode/SPE interface

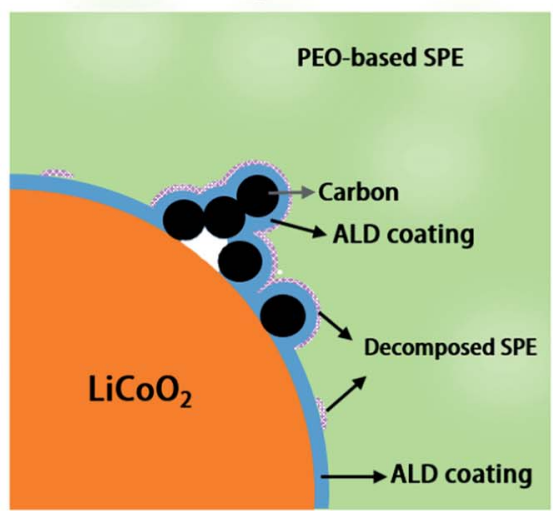

Fig. 1 Schematic diagram showing the working mechanism of ALD coating for enabling stable, high voltage solid polymer electrolyte-based lithium ion batteries. The decomposition of SPES on (a) unprotected $\mathrm{LiCOO}_{2}$ electrode and (b) electrode with protected LiCoO 2 particles (LCOcoating), and the working mechanism of (c) protected electrode (LCO + CB-coating) in ASSLBs after extensive charge/discharge cycles. Conductive carbon can accelerate the decomposition of SPEs at high voltage, and thus, the protection of the carbon/SPE interface is very important for stabilizing high voltage solid-state lithium ion batteries. 
vacuum drying at $60{ }^{\circ} \mathrm{C}$ for 2 days. The obtained $\mathrm{PEO}-\mathrm{LiClO}_{4}-$ LLZTO SPE membrane was immediately transferred to an Arfilled glovebox and rested over 3 days before use.

\section{$\mathrm{LiCoO}_{2}$ electrode preparation}

Bare $\mathrm{LiCoO}_{2}$ electrodes were prepared by mixing $90 \mathrm{wt} \%$ of $\mathrm{LiCoO}_{2}$ particles, $6 \mathrm{wt} \%$ carbon-black (acetylene black) and 4 wt\% poly(vinylidene) fluoride binder in the $N$-methylpyrrolidinone (NMP) solvent. The slurry was coated on $\mathrm{Al}$ foil by a doctor blade method. The electrode was obtained after drying in a vacuum oven at $120{ }^{\circ} \mathrm{C}$ overnight. The loading of $\mathrm{LiCoO}_{2}$ was around $1.5-2 \mathrm{mg} \mathrm{cm}^{-2}$. The obtained bare $\mathrm{LiCoO}_{2}$ electrode was used directly for electrode coating. ALD LTO coatings on $\mathrm{LiCoO}_{2}$ particles or electrodes followed a previously reported ALD procedure from our group. ${ }^{24}$ Briefly, the LTO coatings were deposited using an ALD reactor (Savannah 100, Cambridge Nanotechnology Inc., USA) by alternatively pulsing lithium tertbutoxide, tantalum(v) ethoxide and $\mathrm{H}_{2} \mathrm{O}$ at $235^{\circ} \mathrm{C}$.

\section{Electrochemical performance testing}

All solid-state $\mathrm{LiCoO}_{2}$ batteries were assembled in 2032 type coin cells in an argon-filled glove box (Vacuum Atmosphere Company, moisture and oxygen level less than $1 \mathrm{ppm}$ ). The $\mathrm{LiCoO}_{2}$ electrodes and lithium foil were used as the working electrode and the counter electrode, respectively. The PEO$\mathrm{LiClO}_{4}$-LLZTO SPE was used as both the separator and lithium ion conductor. No additional solvent or liquid electrolyte was applied into the $\mathrm{LiCoO}_{2}$ ASSLBs. Galvanostatic charge/discharge was performed between 2.7 and $4.5 \mathrm{~V}$ in a $60{ }^{\circ} \mathrm{C}$ oven using a LAND battery tester. The ASSLBs were rested at $60^{\circ} \mathrm{C}$ over $30 \mathrm{~h}$ before testing. For $\mathrm{LiCoO}_{2}$ batteries with liquid electrolyte, 2032 type coin cells were assembled with a liquid electrolyte containing 1 M LiPF6 in ethylene carbonate (EC) : ethylmethyl carbonate (EMC) : diethyl carbonate (DEC) solvents with a volume ratio of $1: 1: 1$, using a Celgard separator. Cyclic voltammetry of the $\mathrm{LiCoO}_{2}$ batteries was performed between 2.7 and $4.5 \mathrm{~V}$ (vs. $\left.\mathrm{Li} / \mathrm{Li}^{+}\right)$at $60{ }^{\circ} \mathrm{C}$. Linear sweep voltammetry of $\mathrm{Li} /$ SPE/PEO-carbon cells was conducted at an open-circuit voltage of $4.5 \mathrm{~V}$ on a versatile multichannel potentiostat 3/Z (VMP3). PEO-carbon composite electrodes consisted of $70 \mathrm{wt} \% \mathrm{PEO}$ and 26 wt $\%$ carbon-black (with/without LTO coating) and 4 wt $\%$ $\mathrm{LiClO}_{4}$ was used as the working electrode. Electrochemical impedance spectroscopy (EIS) was performed on a versatile multichannel potentiostat 3/Z (VMP3) by applying an AC voltage with $10 \mathrm{mV}$ amplitude in the frequency range from $500 \mathrm{kHz}$ to $0.01 \mathrm{~Hz}$.

\section{Materials characterization}

The morphology of the samples was characterized using a Hitachi S-4800 field emission scanning electron microscope (FE-SEM) equipped with an energy dispersive X-ray spectrometer (EDX). FIB was conducted in a LEO (Zeiss) 1540XB FIB/SEM machine. Mass spectrometry was conducted on a Shimadzu GCMS-QP2010 gas chromatograph/mass spectrometer. X-ray absorption spectroscopy (XAS) measurements using total electron yield (TEY) and fluorescence yield (FLY) modes at the Co K- edge were performed at the Canadian light source (CLS) at the Soft X-ray Micro-characterization Beamline (SXRMB) with a photon energy range of 1.7-10 keV utilizing a Si (111) crystal monochromator.

\section{Results and discussion}

Cathodes were prepared with $\mathrm{LiCoO}_{2}$ particles, carbon black (acetylene black), and a poly(vinylidene) fluoride binder at a weight ratio of $90: 6: 4$. Using the advanced ALD technique, the lithium tantalate (LTO) coating was deposited on either the $\mathrm{LiCoO}_{2}$ particles before electrode preparation (coating on $\mathrm{LiCoO}_{2}$ active materials, referred to as LCO-coating) or on the electrode surface after casting and drying (coating on the electrode, referred to as LCO + CB-coating) or on the carbon black particles before electrode preparation (coating on CB particles, referred to as CB-coating). Fig. S1a-c $\dagger$ show the scanning electron microscopy (SEM) images of a pristine $\mathrm{LiCoO}_{2}$ electrode, an electrode with ALD LTO coated $\mathrm{LiCoO}_{2}$ particles (LCO-coating), and an ALD LTO coated electrode (LCO + CB-coating). The three electrodes with or without these two types of coatings show a very similar morphology, indicating that the ALD LTO coatings were conformal and non-destructive. The LCO-coating did not alter the distribution of the conductive carbon on the electrode surface, nor did LCO + CB-coating. In addition, from the SEM images, one can easily find that the electrode/SPE interface actually has a large proportion of the conductive carbon/SPE interface since carbon black is uniformly distributed/covered on the $\mathrm{LiCoO}_{2}$ particle surface.

Transmission electron microscopy (TEM), SEM and energydispersive X-ray spectroscopy (EDX) analyses were used to confirm the deposition of the ALD coatings on active materials and the electrode (Fig. 2 and S2-S5 $\dagger$ ). Fig. 2a shows the TEM image of the ALD coating on $\mathrm{LiCoO}_{2}$ particle and the schematic illustration of the LCO-coating electrode is presented in Fig. $2 \mathrm{~b}$. Differently, the ALD LTO coated electrode (LCO + CB-coating) shows a coating on both carbon black and $\mathrm{LiCoO}_{2}$ particles (Fig. 2c). The EDX mapping results in Fig. S3† confirm that ALD LTO is deposited on both the CB and LCO particle surface for the LCO + CB-coating electrode. This type of electrode structure is schematically illustrated in Fig. 2d. Hereby, two types of protective coverages are established: (I) partial protection between the $\mathrm{LiCoO}_{2} / \mathrm{SPE}$ interface only and (II) full protection on the entire electrode covering both $\mathrm{LiCoO}_{2}$ and conductive carbon. The electrochemical performance will be compared systematically.

Cyclic voltammetry (CV) was performed to study the electrochemical process of $\mathrm{LiCoO}_{2}$ ASSLBs with different coatings. For comparison, $\mathrm{CV}$ of a regular $\mathrm{LiCoO}_{2}$ battery with a commercial carbonate-based liquid electrolyte was conducted for a comprehensive understanding. As shown in Fig. S6a, $\uparrow$ the anodic peaks corresponded to the $\mathrm{Li}^{+}$extraction process, and the cathodic peaks were related to the $\mathrm{Li}^{+}$insertion process. There are three pairs of peaks which correspond to three oxidation/reduction couples. The redox pair at $4.07 / 3.74 \mathrm{~V}$ is the result of the conversion between $\mathrm{Co}^{3+}$ and $\mathrm{Co}^{4+}$ for the firstorder phase transformation between two hexagonal phases. ${ }^{25}$ 

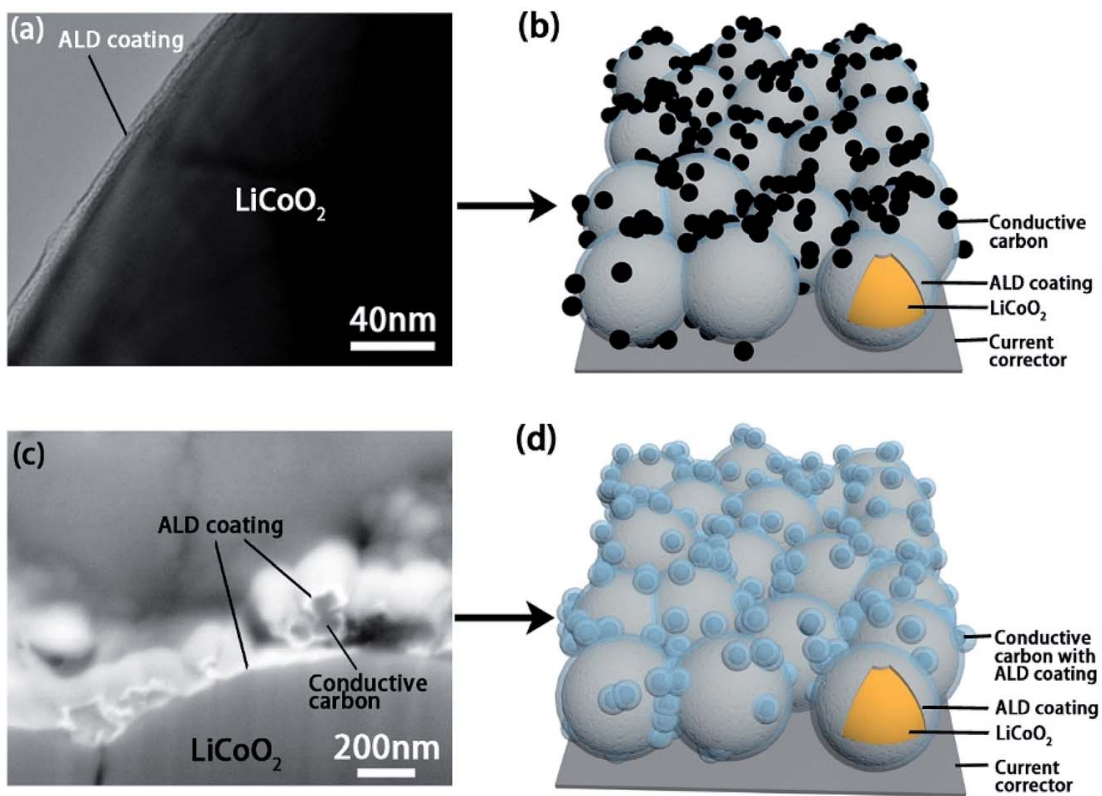

Fig. 2 (a) A TEM image of the 10 cycles of ALD LTO (thickness is $\sim 5 \mathrm{~nm}$ ) coating on $\mathrm{LiCoO}_{2}$ particles and its (b) schematic diagram of the LiCoO 2 electrode with the LCO-coating where conductive carbon is not protected. (c) A SEM image in backscattered electron mode of the 20 cycles of ALD LTO (thickness is $\sim 10 \mathrm{~nm}$ ) coating on both conductive carbon and $\mathrm{LiCoO}_{2}$ particles from the $\mathrm{LCO}+\mathrm{CB}$-coating sample after focused ion beam (FIB) cutting, and its (d) schematic diagram showing the $\mathrm{LiCoO}_{2}$ electrode where both $\mathrm{LiCoO}_{2}$ and conductive carbon are protected. The binder is omitted in the schematic diagrams.

The other two pairs of anodic/cathodic peaks are related to the order-disorder phase transformation between hexagonal and monoclinic phases. ${ }^{26-28}$ In contrast, in ASSLBs, the three pairs of redox peaks are combined into a pair of broad peaks for all three cells with a bare $\mathrm{LiCoO}_{2}$ electrode, electrodes with LCOcoating protection and LCO + CB-coating protection (Fig. S6b$\mathrm{d} \dagger$ ). This is probably because of the sluggish $\mathrm{Li}^{+}$transport in SPEs and electrodes that broadens the peaks related to the firstorder phase transformation reaction, overlapping the other weak peaks. Nevertheless, the ASSLBs with different cathodes still exhibit obvious difference in electrochemical behaviours. For the ASSLB with the bare $\mathrm{LiCoO}_{2}$ electrode, an anodic peak and a cathodic peak are observed with decreasing peak current intensities and increase in overpotentials over the three scans (Fig. S6b $\dagger$ ). This is a typical indication of decay in electrochemical redox reversibility. The decrease in peak current intensities and increase in overpotentials also occur in the ASSLB with LCO-coating protection (Fig. S6c $\dagger$ ). As a conclusion, LCO-coating protection is insufficient in preventing the reversibility decay. In great contrast, instead of a decrease in peak current intensities and increase in overpotentials, the slight increase in peak current intensities and the decrease in overpotentials indicate a more reversible electrochemical process of the LCO + CB-coating protected electrode (Fig. S6d $\dagger$ ).

The electrochemical performances of ASSLBs with different protection coverages were characterized at $60{ }^{\circ} \mathrm{C}$. First, the coating thickness is optimized. Different cycles of ALD LTO were conducted on LCO-coating and LCO + CB-coating electrodes and their results are presented in Fig. S7. $\dagger$ Clearly, the ALD LTO coating thicknesses were optimized to 10 ALD cycles for LCO-coating protection and 20 ALD cycles for LCO + CBcoating protection.

The ASSLBs performances with optimised ALD coating thickness for bare, LCO-coating, and LCO + CB coating are compared in Fig. 3. Consistent with the $\mathrm{CV}$ results, observable voltage polarization along with serious capacity fading is already observed during the first 20 cycles of galvanostatic testing at $0.2 \mathrm{C}$ for both ASSLBs with a bare $\mathrm{LiCoO}_{2}$ electrode (Fig. 3a) or LCO-coating protected electrode (Fig. 3b). This is possibly due to the severe decomposition of PEO-based SPEs at high voltage that increases the interfacial resistance. Even though the LCO-coating protection can protect the $\mathrm{LiCoO}_{2} / \mathrm{SPE}$ interface, the conductive carbon was still in direct contact with the SPE.

In contrast, much smaller polarization increase is observed for the ASSLB with the LCO + CB-coating protected electrode (Fig. 3c). The discharge capacities are also relatively stable compared to the other two cells without protection or only with LCO-coating protection. Thus, the protection on the carbon/SPE interface is evidently very important.

The dramatically different effects between LCO-coating and LCO + CB-coating protections on the electrochemical performance can be further verified by cycling performance and rate performance. Under the optimized conditions, ASSLBs with LCO + CB-coating protection demonstrate substantially enhanced performance over the ASSLBs without protection or with LCO-coating protection (Fig. 3d-f). While all three ASSLBs deliver a similar initial discharge capacity around 170-177 mA h $\mathrm{g}^{-1}$, a retaining capacity of $110.4 \mathrm{~mA} \mathrm{~h} \mathrm{~g}^{-1}$ at 100 th cycles for the ASSLB with the LCO + CB-coating protected electrode is 

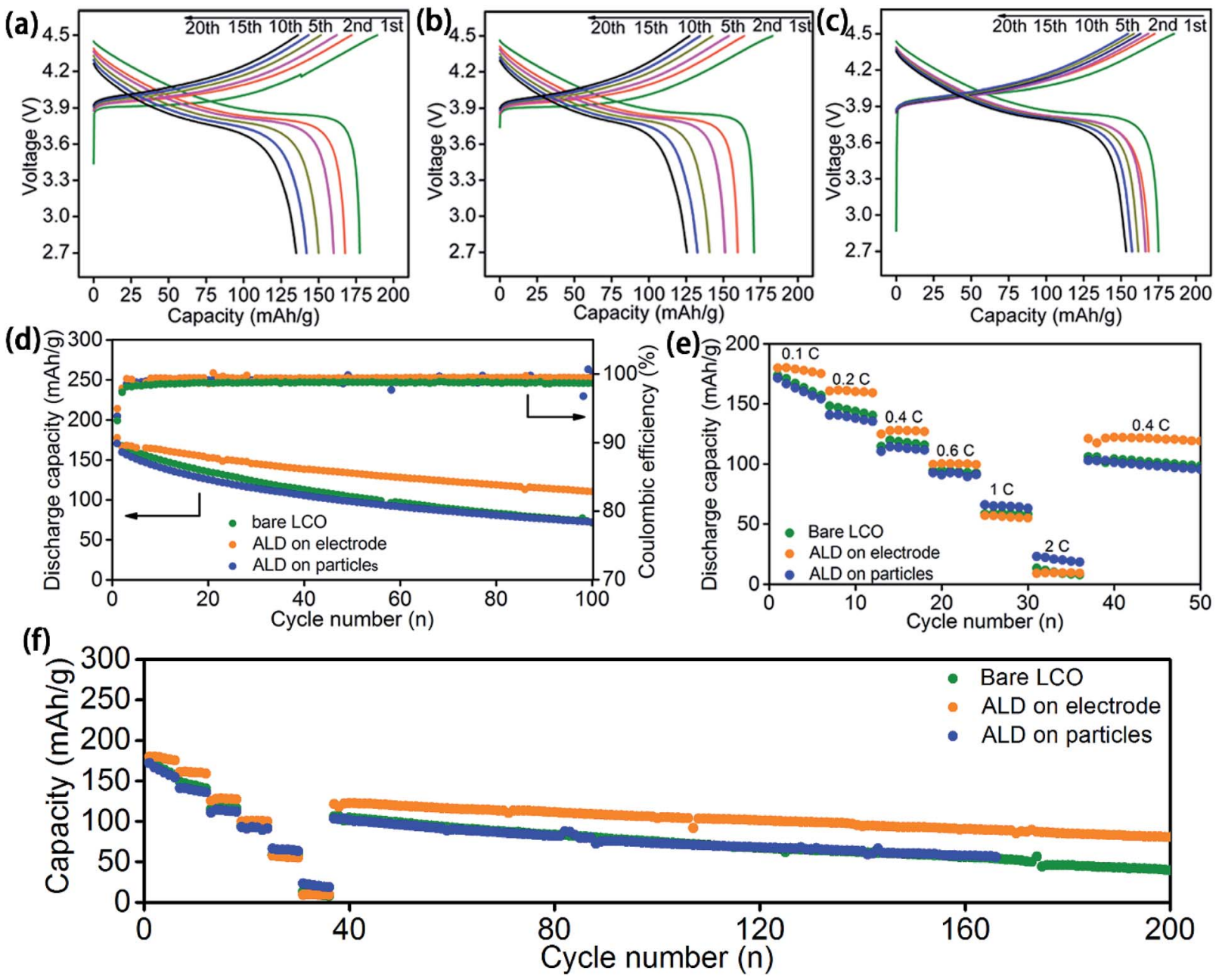

Fig. 3 Charge/discharge profiles of ASSLBs with (a) bare $\mathrm{LiCOO}_{2}$ electrode, (b) LCO-coating protected electrode, and (c) LCO + CB-coating protected electrode at $0.2 \mathrm{C}$ and $60^{\circ} \mathrm{C}$. Corresponding (d) cycling performance, (e) rate performance, and (f) long-term cycling performance after rate performance testing for ASSLBs with different $\mathrm{LiCOO}_{2}$ electrodes at $60{ }^{\circ} \mathrm{C}$.

significantly higher than the remaining capacity of $\sim 71 \mathrm{~mA} \mathrm{~h}$ $\mathrm{g}^{-1}$ for the other two ASSLBs (Fig. 3d). As shown in Fig. 3e, LCO + CB-coating protection also markedly enhanced the rate performance at lower current densities of up to 0.6C. However, at a higher current density, a higher capacity is achieved for the LCO-coating electrode. The reason can be possibly attributed to the higher lithium ion diffusion in the LCO-coating electrode compared to that of the bare and LCO + CB coating electrodes. In the LCO-coating electrode, LCO particles are totally coated with LTO, which is a good lithium ion conductor, while in the LCO + CB-coating protected electrode, only part of LCO particles are exposed for ALD LTO deposition. Therefore, the lithium ion diffusion of the LCO-coating protected electrode is better than that of the LCO + CB-coating protected electrode (Fig. S8 $\dagger$ ) and bare electrode. At high current density, lithium ion diffusion becomes a determining step for the redox reaction. Thus, the electrode with higher lithium ion diffusion can deliver a higher capacity.

LCO + CB-coating protection also can improve the initial coulombic efficiency and subsequent average coulombic efficiency. The initial coulombic efficiencies for the ASSLBs with a bare $\mathrm{LiCoO}_{2}$ electrode, LCO-coating protection, and LCO + CBcoating protection electrodes are 93.8\%, 93.2\%, and 94.9\%, respectively; the subsequent average coulombic efficiencies are $99.0 \%, 98.6 \%$, and $99.3 \%$ in order. The LCO-coating protection electrode actually shows barely any improvement over the bare $\mathrm{LiCoO}_{2}$ electrode, indicating the protection of only $\mathrm{LiCoO}_{2}$ particles is not enough in PEO-based ASSLBs. The higher coulombic efficiency by LCO + CB-coating protection indicates reduced decomposition of the PEO-based SPE during high voltage charge/discharge cycles in ASSLBs.

Electrochemical impedance spectroscopy (EIS) was conducted to study the evolution of interfacial resistance in the $\mathrm{LiCoO}_{2}$ ASSLBs upon cycling (Fig. 4). The three ASSLBs without coating or with LCO-coating or with LCO + CB-coating present EIS spectra with a similar shape but different evolution trends. There are two semicircles with one at high frequency and one at low frequency. Fig. 4d shows the equivalent circuit for fitting these EIS spectra. $R_{\mathrm{e}}$ is the impedance from the solid polymer electrolyte, $R_{\mathrm{a}}$ is the interfacial impedance between the lithium 

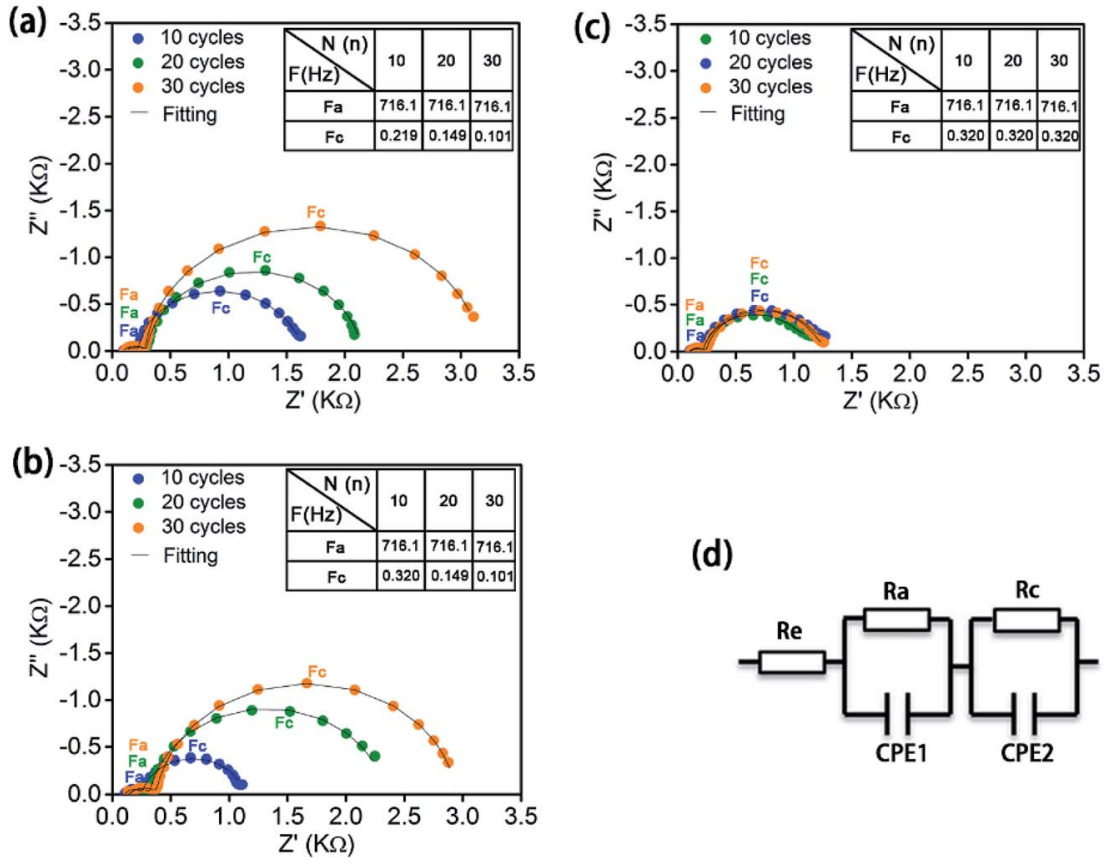

(d)

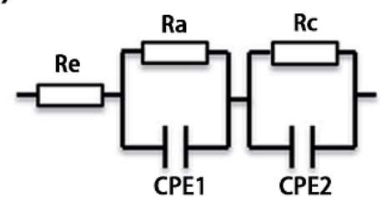

Fig. 4 EIS spectra of ASSLBs with (a) bare $\mathrm{LiCoO}_{2}$ electrode, (b) $\mathrm{LCO}$-coating protected $\mathrm{LiCoO}_{2}$ electrode, and (c) LCO + CB-coating protected $\mathrm{LiCOO}_{2}$ electrode after 10, 20, and 30 cycles of charge/discharge. The inset tables show the critical frequency values on top of the high frequency semi-circle $\left(F_{\mathrm{a}}\right)$ and low frequency semi-circle $\left(F_{\mathrm{c}}\right)$ at different cycles of charge/discharge, respectively. (d) The equivalent circuit for fitting the EIS spectra.

anode and SPE, and CPE1 is its corresponding constant phase element. $R_{\mathrm{c}}$ is the interfacial impedance between the cathode and SPE, and CPE2 is its corresponding constant phase element. ${ }^{18}$ All ASSLBs show relatively stable anode/SPE interfacial resistance with similar values, while dramatic difference is present at the cathode/SPE interfaces. For the bare $\mathrm{LiCoO}_{2}$ ASSLB (Fig. 4a), a significant increase in the cathode/SPE interfacial resistance is observed over 30 cycles, enlarging from $1338 \Omega$ at the 10 th cycle to $1843 \Omega$ at the 20 th cycle to $2891 \Omega$ at the 30th cycle. Similarly, consistent with the electrochemical performance, a significant increase in the cathode/SPE interfacial resistance is also observed in the ASSLB with LCO-coating protection (Fig. 4b). The increase in cathode/SPE interfacial resistance can be attributed to the accumulation of insulating SPE decomposition products, which can block the $\mathrm{Li}^{+}$ion transportation at the interface. In contrast, the cathode/SPE interfacial impedance of the ASSLB with LCO + CB-coating protection is stabilized to $\sim 920 \Omega$ after 30 cycles, confirming the stable SPE/cathode interface enabled by the ALD LTO coating on the electrode. The EIS results strongly support the superior effects of ALD LTO protection for high-voltage ASSLBs.

To confirm that the conductive carbon/SPE interface is important for the ASSLB performance enhancement, an ASSLB with a CB-coated electrode (an electrode with bare $\mathrm{LiCoO}_{2}$ and LTO coated CB) was assembled and studied. The results are presented in Fig. S10.† It shows that LTO coating on carbon particles can also enhance the cycling performance of the ASSLB, and its cycling performance is very similar to that of LCO + CB coated ASSLBs.
Why is the conductive carbon/SPE interface so critical? It is well known that the electrochemical oxidation potential of PEObased SPEs is around $3.8 \mathrm{~V}$ vs. $\mathrm{Li} / \mathrm{Li}^{+}$according to the linear sweep voltammetry method using a PEO-carbon composite electrode. ${ }^{10}$ Conventionally, it has been reported that electronic conductive additives (usually carbon black) play an important role in realizing completely utilization of electrode active materials for batteries, because the occurrence of electrochemical reactions requires both electrons and $\mathrm{Li}^{+}$ions. However, in ASSLBs with $\mathrm{Li}^{+}$ion conductive SSEs, the presence of conductive carbons could also trigger irreversible decomposition of the SSEs, ${ }^{23}$ leading to low coulombic efficiency and performance decay.

Even conductive carbon black particles occupy a low weight content in the electrodes. The large volume of carbon black and resulted the large proportion of the carbon/SPE interface among the cathode/SPE interface cannot be neglected (Fig. S1a-c $\dagger$ ). This could explain the similar performance of ASSLBs with the bare $\mathrm{LiCoO}_{2}$ electrode and LCO-coating electrode, whereas the carbon black particles are in direct contact with the SPE in either case. The linear sweep voltammetry results of carbon-SPE composite electrodes with or without ALD LTO coating also confirmed the importance of the carbon/SPE interface. As shown in Fig. 5a, the overshooting current approaching $4.5 \mathrm{~V}$ is observed for the bare carbon-PEO electrode, which indicates the serious decomposition of PEO at $4.5 \mathrm{~V}$. In contract, this overshooting current is significantly reduced by the ALD LTO coated conductive carbon. As supported by the mass spectrometry results in Fig. $\mathrm{S} 11 \mathrm{~b}, \uparrow$ the significant signals from decomposed SPE products are presented in the bare carbon- 

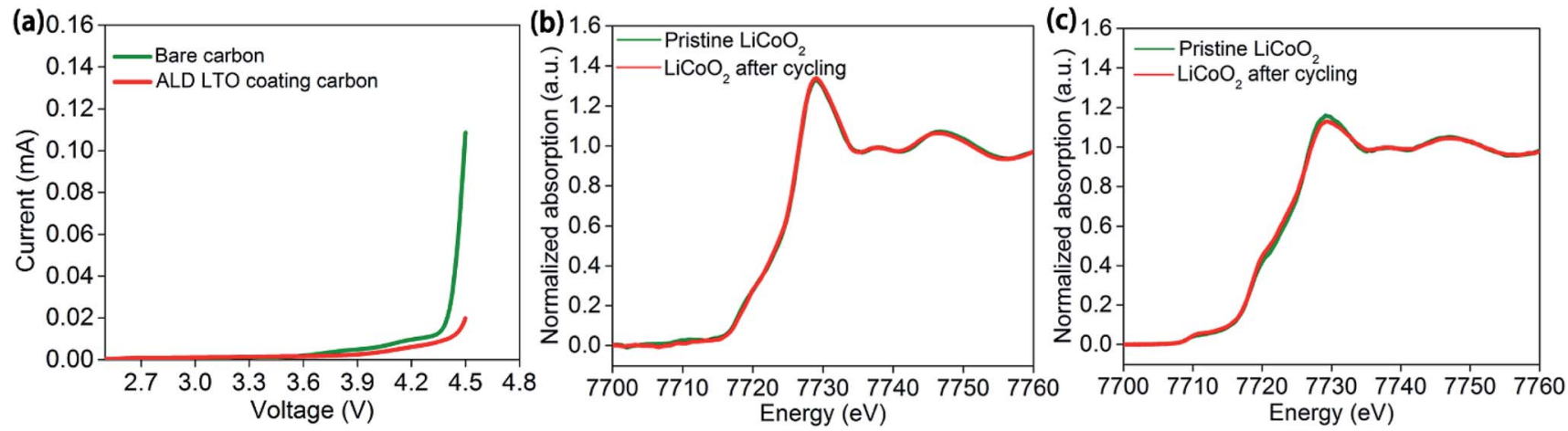

Fig. 5 (a) Comparison of the linear sweep voltammogram of the Li/SPE/PEO-carbon composite cell and Li/SPE/PEO-LTO@carbon composite cell (scan rate $=0.3 \mathrm{mV} \mathrm{s}^{-1}$, from OCV to $4.5 \mathrm{~V} \mathrm{vs}$. $\mathrm{Li}_{/} / \mathrm{Li}^{+}$). Comparison of the XAS Co K-edge spectra of unprotected $\mathrm{LiCoO} 2$ before and after 5 cycles of charge/discharge in ASSLBs in the discharge state in terms of (b) TEY mode and (c) FLY mode.

PEO electrode. However, with the ALD LTO coating on carbon, the signals from the decomposed SPE are reduced (Fig. S11c $\dagger$ ), which demonstrates the excellent capability of LTO in stabilizing the carbon/SPE interface at high voltage. This may be the reason why the protection of the conductive carbon/SPE interface can achieve such a significant enhancement in electrochemical performance.

Why does the LCO-coating electrode show almost no enhancement? It is possible that $\mathrm{LiCoO}_{2}$ active materials are stable and further ALD protection cannot enhance their stability in our study. This conclusion is supported by the X-ray absorption spectroscopy (XAS) of the unprotected $\mathrm{LiCoO}_{2}$ electrodes before and after cycling with the PEO-based SPE. The Co K-edge XAS results for $\mathrm{LiCoO}_{2}$ electrodes before and after 5 charge/discharge cycles at the discharge state in ASSLBs showed almost no difference under both total electron yield (TEY) mode (Fig. 5b) and fluorescence yield (FLY) mode (Fig. 5c). TEY mode provides surface chemical information up to several nanometers, and FLY mode reveals bulk chemical information. The XAS results indicated that the $\mathrm{LiCoO}_{2}$ active materials are relatively stable from the surface to bulk upon cycling in PEO-based ASSLBs. This is also supported by the stable cycling performance of the $\mathrm{LiCoO}_{2}$ battery with a carbonate-based liquid electrolyte (Fig. S12†). Since $\mathrm{LiCoO}_{2}$ is stable during change/discharge processes, it is reasonable that the ALD LTO coating on $\mathrm{LiCoO}_{2}$ particles (LCOcoating protection) showed little effect while the ALD LTO coating on the electrode (LCO + CB-coating protection) achieved significant enhancement.

\section{Conclusions}

The effects of ALD lithium tantalate coatings on cathode active material particles, carbon black particles and the electrode surface were studied systematically for enhancing the highvoltage performance of PEO-based ASSLBs. Using stable $\mathrm{LiCoO}_{2}$ particles as an example for a high voltage cathode up to $4.5 \mathrm{~V}$, we demonstrated the importance of the protection on the conductive carbon/SPE interface. It is found that interfacial protection covering the carbon/SPE interface is very important to stabilize the PEO-based SPE at high voltage and enhance the cycling performance of ASSLBs. Significantly improved cycling performance and rate performance were demonstrated in ASSLBs with simple 20 ALD cycles of lithium tantalate coating on the electrode surface. This study sheds light on the rational design of protective coatings for the polymer electrolyte-electrode interface to enhance the high voltage performance of ASSLBs.

\section{Statement of contributions}

J. Liang, Q. Sun and X. Sun conceived the idea and experiments; J. Liang conducted the electrode/SPE preparations, electrochemical performance testing, and SEM. Y. Zhao, F. Zhao and Y. Sun helped to conduct the ALD coating experiment. Y. Sun assisted in the synchrotron XAS measurement; J. Luo, X. Li, R. $\mathrm{Li}$, X. Lin, L. Zhang, S. Lu and H. Huang participated in data analysis and discussion; J. Luo polished the manuscript; X. Sun supervised the overall project. All authors discussed the results and commented on the manuscript.

\section{Conflicts of interest}

There are no conflicts to declare.

\section{Acknowledgements}

This work was supported by China Automotive Battery Research Institute, the Natural Sciences and Engineering Research Council of Canada (NSERC), the Canada Research Chair Program (CRC), the Canadian Light Source (CLS) and the University of Western Ontario. Jianneng Liang greatly appreciates the China Scholarship Council (CSC) for supporting this study. Qian Sun appreciates the support of MITACS Elevate postdoctoral program.

\section{Notes and references}

1 A. Manthiram, X. Yu and S. Wang, Nat. Rev. Mater., 2017, 2, 16103. 
2 C. Sun, J. Liu, Y. Gong, D. P. Wilkinson and J. Zhang, Nano Energy, 2017, 33, 363-386.

3 J. Liang, Q. Sun, Y. Zhao, Y. Sun, C. Wang, W. Li, M. Li, D. Wang, X. Li and Y. Liu, J. Mater. Chem. A, 2018, 6, 23712-23719.

4 J. Liang, J. Luo, Q. Sun, X. Yang, R. Li and X. Sun, Energy Storage Materials, 2019, 21, 308-334.

5 V. Thangadurai, S. Narayanan and D. Pinzaru, Chem. Soc. Rev., 2014, 43, 4714-4727.

6 L. Long, S. Wang, M. Xiao and Y. Meng, J. Mater. Chem. A, 2016, 4, 10038-10069.

7 F. Croce, G. Appetecchi, L. Persi and B. Scrosati, Nature, 1998, 394, 456.

8 D. Lin, W. Liu, Y. Liu, H. R. Lee, P.-C. Hsu, K. Liu and Y. Cui, Nano Lett., 2015, 16, 459-465.

9 K. J. Harry, D. T. Hallinan, D. Y. Parkinson, A. A. MacDowell and N. P. Balsara, Nat. Mater., 2014, 13, 69.

10 Y. Xia, T. Fujieda, K. Tatsumi, P. P. Prosini and T. Sakai, J. Power Sources, 2001, 92, 234-243.

11 K. K. Fu, Y. Gong, J. Dai, A. Gong, X. Han, Y. Yao, C. Wang, Y. Wang, Y. Chen and C. Yan, Proc. Natl. Acad. Sci. U. S. A., 2016, 113, 7094-7099.

12 J. Zhang, N. Zhao, M. Zhang, Y. Li, P. K. Chu, X. Guo, Z. Di, X. Wang and H. Li, Nano Energy, 2016, 28, 447-454.

13 C. Yang, B. Liu, F. Jiang, Y. Zhang, H. Xie, E. Hitz and L. Hu, Nano Res., 2017, 10, 4256-4265.

14 C.-Z. Zhao, X.-Q. Zhang, X.-B. Cheng, R. Zhang, R. Xu, P.-Y. Chen, H.-J. Peng, J.-Q. Huang and Q. Zhang, Proc. Natl. Acad. Sci. U. S. A., 2017, 114, 11069-11074.

15 P. Hovington, M. Lagacé, A. Guerfi, P. Bouchard, A. Mauger, C. Julien, M. Armand and K. Zaghib, Nano Lett., 2015, 15, 2671-2678.
16 L. Wang, B. Chen, J. Ma, G. Cui and L. Chen, Chem. Soc. Rev., 2018, 47, 6505-6602.

17 K. Nie, Y. Hong, J. Qiu, Q. Li, X. Yu, H. Li and L. Chen, Front. Chem., 2018, 6, 616.

18 S. Seki, Y. Kobayashi, H. Miyashiro, Y. Mita and T. Iwahori, Chem. Mater., 2005, 17, 2041-2045.

19 H. Miyashiro, Y. Kobayashi, S. Seki, Y. Mita, A. Usami, M. Nakayama and M. Wakihara, Chem. Mater., 2005, 17, 5603-5605.

20 J. Ma, Z. Liu, B. Chen, L. Wang, L. Yue, H. Liu, J. Zhang, Z. Liu and G. Cui, J. Electrochem. Soc., 2017, 164, A3454A3461.

21 Q. Yang, J. Huang, Y. Li, Y. Wang, J. Qiu, J. Zhang, H. Yu, X. Yu, H. Li and L. Chen, J. Power Sources, 2018, 388, 65-70.

22 F. Han, Y. Zhu, X. He, Y. Mo and C. Wang, Adv. Energy Mater., 2016, 6, 1501590.

23 W. Zhang, T. Leichtweiß, S. P. Culver, R. Koerver, D. Das, D. A. Weber, W. G. Zeier and J. r. Janek, ACS Appl. Mater. Interfaces, 2017, 9, 35888-35896.

24 J. Liu, M. N. Banis, X. Li, A. Lushington, M. Cai, R. Li, T.-K. Sham and X. Sun, J. Phys. Chem. C, 2013, 117, 2026020267.

25 Y. Gu, D. Chen, X. Jiao and F. Liu, J. Mater. Chem., 2007, 17, 1769-1776.

26 K. Dokko, S. Horikoshi, T. Itoh, M. Nishizawa, M. Mohamedi and I. Uchida, J. Power Sources, 2000, 90, 109-115.

27 J. N. Reimers and J. Dahn, J. Electrochem. Soc., 1992, 139, 2091-2097.

28 X. Li, J. Liu, X. Meng, Y. Tang, M. N. Banis, J. Yang, Y. Hu, R. Li, M. Cai and X. Sun, J. Power Sources, 2014, 247, 57-69. 